\title{
Dynamic Energy Balance and Obesity Prevention
}

\author{
Sunmi Yoo* \\ Department of Family Medicine, Inje University Haeundae Paik Hospital, Busan, Korea
}

Dynamic energy balance can give clinicians important answers for why obesity is so resistant to control. When food intake is reduced for weight control, all components of energy expenditure change, including metabolic rate at rest (resting energy expenditure [REE]), metabolic rate of exercise, and adaptive thermogenesis. This means that a change in energy intake influences energy expenditure in a dynamic way. Mechanisms associated with reduction of total energy expenditure following weight loss are likely to be related to decreased body mass and enhanced metabolic efficiency. Reducing calorie intake results in a decrease in body weight, initially with a marked reduction in fat free mass and a decrease in REE, and this change is maintained for several years in a reduced state. Metabolic adaptation, which is not explained by changes in body composition, lasts for more than several years. These are powerful physiological adaptations that induce weight regain. To avoid a typically observed weight-loss and regain trajectory, realistic weight loss goals should be established and maintained for more than 1 year. Using a mathematical model can help clinicians formulate advice about diet control. It is important to emphasize steady efforts for several years to maintain reduced weight over efforts to lose weight. Because obesity is difficult to reverse, clinicians must prioritize obesity prevention. Obesity prevention strategies should have high feasibility, broad population reach, and relatively low cost, especially for young children who have the smallest energy gaps to change.

Key words: Energy metabolism, Body composition, Prevention, Obesity

\author{
Received August 21, 2018 \\ Reviewed October 3, 2018 \\ Accepted October 5, 2018 \\ ${ }^{*}$ Corresponding author \\ Sunmi Yoo \\ https://orcid.org/0000-0001-7407-8423 \\ Department of Family Medicine, Inje \\ University Haeundae Paik Hospital, \\ 875 Haeun-daero, Haeundae-gu, \\ Busan 48108 , Korea \\ Tel: +82-51-797-3220 \\ Fax: +82-51-797-2451 \\ E-mail:syoo@paik.ac.kr
}

\section{INTRODUCTION}

Overweight and obesity have been increasing worldwide for several decades. In 2015, more than 100 million children and 600 million adults worldwide were obese. ${ }^{1}$ Obesity accounted for about four million deaths and 120 million disability-adjusted life-years worldwide in 2015. ${ }^{1}$ While some developed countries have seen a slowing in the rise in obesity prevalence since mid-2000, present interventions and policies have not been able to stop the rise in body mass index (BMI) in most countries. ${ }^{2}$

Lifestyle interventions such as healthy diet and increased physical activity have been widely recommended to reduce the risk of obesity-related complications as well as obesity itself. While these interventions are the basis of treatment for obesity, it is hard to demonstrate that they can effectively manage obesity. Although several medications approved for chronic weight management can be useful for patients who have been unsuccessful with diet and exercise alone, bariatric surgery may be the most effective intervention for weight loss, disease prevention, and remission thus far. ${ }^{3}$ However, long-term health outcomes and safety related to bariatric surgery are still unclear, and the high cost and health system barriers hinder most people. If we are to effectively prevent and treat obesity, it is essential to rethink why obesity is so resistant to treatment and to gain a better understanding of energy homeostasis, which is one of the reasons why voluntary weight loss is so difficult to achieve and maintain.

The aims of this narrative review are (1) to understand recent research results about dynamic energy balance, (2) to apply this research to clinical practice of obesity treatment in terms of body compositional change, and (3) to estimate the change in dynamic 
energy balance for obesity control.

\section{DYNAMIC ENERGY BALANCE}

\section{Static versus dynamic energy balance}

Reducing energy intake for weight control causes a negative energy balance, which means that energy intake is less than energy expenditure. There are two views on the relationship of the sides of the energy balance equation. Static or linear energy balance simply assumes that a change in energy intake does not change or influence energy expenditure. On the other hand, dynamic or nonlinear energy balance assumes that numerous biological and behavioral factors regulate and influence both sides of the energy balance equation. Thus, a change in factors related to energy intake can and does influence factors related to energy expenditure. In reality, reducing food intake for weight control changes all aspects of energy expenditure, including metabolic rate at rest, metabolic rate of exercise, and adaptive thermogenesis (AT).

A typical misconception associated with static energy balance is the so-called 3,500-kcal rule. One pound of fat corresponds to energy of about 3,500 kcal, and the "3500-kcal rule" claims that, if a negative energy balance of 3,500 kcal is created by eating less and exercising more for 1 week, weight is slowly and steadily reduced by 1 pound of body fat. When converted to a standard unit, $1 \mathrm{~kg}$ of fat corresponds to 7,200 kcal; thus, a negative energy balance of $500 \mathrm{kcal} /$ day could potentially reduce body fat by $1 \mathrm{~kg}$ in 2 weeks. This measure has been continuously cited as a "rule of thumb" on weight loss educational websites for the lay population ${ }^{4}$ as well as in textbooks ${ }^{5}$, scientific papers ${ }^{6}$, and expert guidelines. ${ }^{3}$ However, when data from real-world weight loss studies were applied, the 3,500-kcal rule overestimated weight loss in most cases. ${ }^{7}$ The actual weight loss pattern is not linear as the 3,500-kcal rule indicates and has different configurations depending on age, sex, height, body composition, etc. On the other hand, the dynamic models result in a curvilinear pattern of weight loss over time and depend on age, sex, height, baseline weight, and degree of caloric restriction, according to the first law of thermodynamics.?

Recently, several mathematical models of dynamic energy balance have helped more accurately predict body weight changes in response to changes in energy intake and expenditure. They in- clude a model developed by Hall et al. ${ }^{8}$ at the National Institutes of Health ${ }^{9}$ and a model developed by Thomas et al. ${ }^{10}$ at the Pennington Biomedical Research Center. ${ }^{11}$ Each model simulates how alterations in energy deficit or excess will affect body weight over a given time period.

For example, the model developed by Hall et al. ${ }^{8}$ predicted body weight according to an effort to reduce it over a 10-year simulation. If $100 \mathrm{~kg}$, sedentary male follows a step reduction of energy intake by $500 \mathrm{kcal} /$ day, his weight is predicted to remain at about $75 \mathrm{~kg}$ for a 10-year simulation. About half of that weight loss would occur in the first year, and about $95 \%$ would be achieved within 3 years. However, the popular 3,500-kcal rule predicts that the same 500 $\mathrm{kcal} /$ day reduction of energy intake will result in a linear decrease of body weight over time, with $22 \mathrm{~kg}$ lost in the first year. ${ }^{8}$ If the dynamic changes of energy expenditure with weight loss are ignored, the magnitude of the error can reach about $10 \mathrm{~kg}$ in the first year.

It is the metabolically active component of the body, i.e., fat-free mass (FFM), that declines or increases in energy expenditure occur. Most of these changes take place passively and are nonadaptive. However, as mentioned earlier, weight change does not exactly follow the prediction based on calculation of energy imbalance. This is explained by FFM-independent metabolic adaptations, i.e., AT that limits weight changes when food intake is reduced or energy consumption is increased.

The total energy expenditure (TEE) of the human body is composed of resting energy expenditure (REE), that is, energy needed to fuel cellular function; non-REE, or energy expended during physical activity; and diet-induced thermogenesis (DIT), or the thermal effect of feeding, that is, the energy needed to process ingested food. Non-REE is an activity-related component including exercise and non-exercise activity thermogenesis. AT refers to changes in REE and non-REE, which are independent from changes in FFM and its composition. ${ }^{12}$ In the next section, the adaptive changes of the human body after caloric restriction are examined, with consideration for body composition.

\section{Dynamic energy balance compensates for long-term weight gain \\ Individuals with normal weight maintain their energy balance over a period of time in a remarkably accurate way. For example, a}


healthy adult weighing $75 \mathrm{~kg}$ typically consumes $3,000 \mathrm{kcal}$ every day (i.e., nearly 1 million kilocalories each year), and a mismatch of just 1\% (i.e., expending 10,950 fewer kilocalories per year or 30 kcal fewer per day than are consumed) will yield a body fat increase of $1.1 \mathrm{~kg}$ after 1 year (considering that the energy content per kilogram change of body fat is $9,440 \mathrm{kcal}$ ). Therefore, energy intake and expenditure must be balanced with greater than $99 \%$ accuracy for those who show smaller changes in weight on an annual basis. ${ }^{13}$ In reality, based on day-to-day experience, most individuals eat more on some days than others and are more active on some days than others. One study demonstrated that recording food intake for 2 weeks revealed interesting findings; compensatory intake occurred with a lag time of 3 to 4 days when more than the average energy intake was consumed. ${ }^{14}$ The existence of corrective mechanisms for smoothing out the day-to-day variation of energy intake suggests that small changes in caloric intake associated with modification of food items are unlikely to have a significant impact.

It is also possible to examine the trend of food intake over a long period of time. The Food and Agriculture Organization of the United Nations calculated that the average American consumes more than $3,600 \mathrm{kcal}$ a day, or a total of 1.3 million kcal a year. ${ }^{15}$ The U.S. Department of Agriculture calculates that a sedentary adult male requires $2,200 \mathrm{kcal}$ a day, or $803,000 \mathrm{kcal}$ a year, and those who are physically active require $2,800 \mathrm{kcal}$ a day, an annual intake of 1.02 million kcal. Without the intervention of compensatory mechanisms, this significant excess of energy intake over expenditure would result in a massive increase in body weight every year. Therefore, the mismatch in energy balance on a day-to-day basis is corrected by the energy homeostasis system over long periods of time. ${ }^{16}$

Two models have dominated the discussion regarding the mechanisms by which body weight is regulated between energy intake and expenditure. The set point model suggests that there is an active feedback mechanism linking adipose tissue (stored energy) to food intake and expenditure via a set point, presumably encoded in the brain. Obesity occurs through the following two related but distinct processes: (1) sustained positive energy balance (energy intake > energy expenditure) and (2) increasing the set point. The latter process explains why weight lost through changes in diet and/or lifestyle tends to be regained over time, which is a major obstacle to effective obesity treatment. ${ }^{13}$ This model is consistent with many of the biological aspects of energy balance, but does not effectively explain the global obesity epidemic of the past 30 years.

On the other hand, the settling point model suggests that body weight is associated with patterns of food intake and physical activity into which people "settle" over time. The biological mechanisms that control energy balance are programmed by environmental factors, and the point at which body weight is maintained may change. In this system, the level of the energy reservoir (fat stores) settles to an equilibrium that is determined by inflow (food intake), which is matched to outflow (energy expenditure), as the rate of outflow is passively related to the level of the reservoir. Hence, the settling point model explains the increasing prevalence of obesity as a result of food availability, more exposure to food signals (i.e., higher food intake), or a reduction of physical activity needs, so-called obesogenic environment. ${ }^{17}$

There is no doubt that changes in the availability of food and its increased caloric content have played a major role in the obesity epidemic. However, public health advice to reduce food intake has not provided a fundamental solution in resolving obesity. Although high calorie intake was an important cause of the problem initially, its reduction is not able to solve it. Reducing calorie intake results in a decrease in body weight, followed by strong physiological adaptations to regain weight. ${ }^{18}$ When initial attention to attempting weight loss decreases or disappears, body weight increases and returns to or even exceeds the original level. ${ }^{16}$

\section{BODY COMPOSITION CHANGE AFTER NEGATIVE ENERGY BALANCE}

\section{Short-term adaptation after weight loss}

According to dynamic energy balance, weight loss results from a negative energy balance and changes in body composition (Fig. 1). It is not continuous but ends in a curvilinear way when a new equilibrium between energy intake and energy expenditure is reached. ${ }^{19}$ Changes in body composition during weight loss are related to AT. During caloric restriction, the first phase of weight loss is rapid and lasts from a few days to a month (phase 1); this is followed by a second phase characterized by a slower weight loss (phase 2). ${ }^{19}$ With caloric restriction, negative energy balance and weight loss 


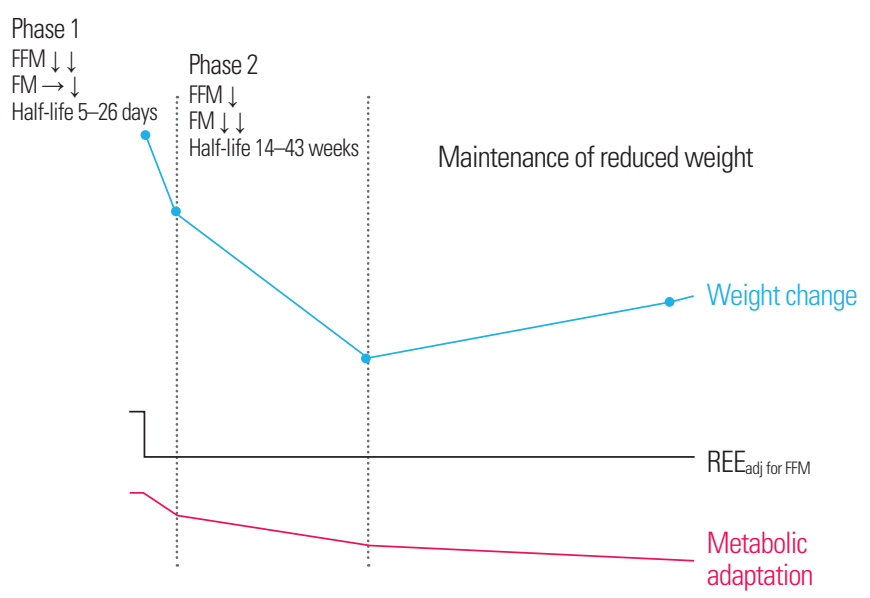

Figure 1. Overview of metabolic adaptation of the typical outpatient weight loss and regain trajectory. During the first few days to weeks of caloric restriction, metabolic adaptation occurs in the resting energy expenditure (REE). In this period, weight decreases rapidly in the fat-free mass (FFM) due to the combined effects of glycogen, protein, and fluid loss. On the other hand, fat mass (FM) slowly decreases during the period of subsequent caloric restriction. Behavioral and metabolic adaptation of dieters contributes to the typical outpatient weight plateau and regain trajectory. Adaptive thermogenesis is characterized by an adaptation of the REE, which is maintained throughout further weight loss and during successful maintenance of reduced body weight. Metabolic adaptation persists over several years after weight loss.

cause reductions in all the energy expenditure components, i.e., REE, DIT, and activity-related energy expenditure. The entire change of energy expenditure is the AT, and it varies between 100 and $500 \mathrm{kcal} /$ day. AT is observed in lean as well as overweight subjects, independent of the weight loss strategy. ${ }^{12}$

During phase 1, more FFM decreases than fat mass. During the first week of caloric restriction, hepatic glycogen stores are depleted due to an immediate drop in insulin secretion, resulting in natriuresis and a reduction in extracellular water. A negative nitrogen balance from protein loss results primarily from gastrointestinal tract and liver proteins involved with nutrient processing in the early phase of weight loss. Later, proteolysis occurs in skeletal muscle and other visceral organs. Thus, water associated with protein catabolism is an important contributor to the rapid weight loss observed in phase 1 . The combined effects of glycogen, protein, and fluid loss largely account for the rapid weight loss of phase 1 compared with the slower rates observed in phase 2 . The rapid weight loss phenomenon during phase 1 is the basis for countless "quick fix" diets such as "one food diets." ${ }^{19}$ Cell mass per se does not decrease, but if the negative energy balance continues, the fat cells decrease up to a new steady state. ${ }^{12}$
According to a caloric restriction study, reductions in fat mass explained $34 \%$ of weight loss during phase 1 , but this proportion increased to $54 \%$ and $68 \%$ during phase 2 . Results indicate that regulation of AT occurred mainly during phase 1 because the AT became manifest within the first 3 days after caloric restriction, with no further changes during phase 2 . After a decrease in body weight, the AT decreased with leptin reduction due to body fat depletion. TEE also remained lower due to reduced AT in the nonREE; it was accompanied by increased skeletal muscle work efficiency and decreased plasma levels of leptin and T3 associated with low sympathetic nervous system activity. ${ }^{20}$ All of these endocrine changes inevitably carried the risk of weight regain.

\section{Changes of body composition and energy expenditure} after rapid, dramatic weight loss

It is possible to obtain important information about changes in body composition and energy expenditure after dramatic weight loss from the results of an American competition reality show. From 2004 to 2016, “The Biggest Loser” featured obese or overweight contestants competing to win a cash prize by seeing who could lose the highest percentage of weight relative to their initial weight. Participants were encouraged to consume a calorie-restricted diet greater than $70 \%$ of their baseline energy requirements and to perform vigorous exercise more than 90 minutes per day for 3 months. After that, it was recommended that they continue eating and exercise interventions at the same level back at home. At week 30, all participants were tested for body composition and energy expenditure, i.e., FM, REE, and TEE. On average, participants lost more than one-third of their initial body weight (initial mean BMI, $49.4 \mathrm{~kg} / \mathrm{m}^{2}$; mean BMI at 30 weeks, $30.4 \mathrm{~kg} / \mathrm{m}^{2}$ ), $83 \%$ from fat and $17 \%$ from FFM. Participants who rapidly lost a significant amount of weight through diet restriction and vigorous physical activity preserved much of their FFM. Relative preservation of FFM was likely due to maintenance or possible increase of skeletal muscle tissue during vigorous exercise.

However, REE was reduced by $789 \mathrm{kcal} /$ day, which was $504 \mathrm{kcal} /$ day greater than expected, based on changes in body weight and composition. A substantial decrease in resting metabolism during active weight loss might not be avoided by the addition of an exercise program. FFM preservation did not prevent slowing of the meta- 
bolic rate during active weight loss; therefore, participants tended to regain weight unless they maintained high levels of physical activity and/or significant caloric restriction..$^{21}$ Long-term change of resting metabolism and metabolic adaptation is more intriguing. Suppression of resting metabolism continued after 6 years of competition, despite substantial weight regains. On the other hand, the magnitude of metabolic adaptation increased 6 years after the competition. ${ }^{22}$ Considering that study subjects maintained a similar level of physical activity over a 6-year period, weight regain due to decreased REE and increased metabolic adaptation after massive weight loss seem to be inevitable. Therefore, long-term weight loss requires vigilant combat against persistent metabolic adaptation that acts to proportionally counter ongoing efforts to reduce body weight.

Analysis using the computational model of metabolism by Hall et $\mathrm{al} .{ }^{8}$ showed that participants could perform an average energy intake of 3,000 kcal/day and $20 \mathrm{~min} /$ day of vigorous activity to maintain their weight loss. However, when individuals returned to their original diet of $3,700 \mathrm{kcal} /$ day and a sedentary lifestyle, weight gain was simulated at a slow rate. ${ }^{23}$

Metabolic adaptation occurs in energy expenditure even when individuals attempt to lose weight by exercise alone without diet control. When overweight or obese women $(n=30$; mean BMI, $30.6 \mathrm{~kg} / \mathrm{m}^{2}$ ) tried to lose weight only with supervised aerobic exercise for 12 weeks, $43 \%$ of participants experienced metabolic adaptation and a greater-than-expected decline in REE $(-102.9 \pm$ $77.5 \mathrm{kcal} /$ day). Thus, both energy expenditure and energy intake were affected by adaptive metabolic response to weight loss due to exercise. $^{18}$

Thus, weight loss, whether diet-induced or exercise-driven, leads to decrease in TEE, REE, and non-REE. Mechanisms associated with reduction of TEE following weight loss are likely to be related to decreased body mass and enhanced metabolic efficiency.

\section{ESTIMATION OF ENERGY BALANCE FOR OBESITY CONTROL}

\section{Estimation for obesity treatment at the individual level}

Using a mathematical model that reflects dynamic energy balance, it is possible to make personalized calorie and physical activi- ty plans to reach a goal weight within a specific time period and to maintain it afterward. Fig. 2 shows an example of a weight change and maintenance phase program. It is a screen-shot of a web-based model simulating the required reduction of dietary energy intake by $385 \mathrm{kcal} /$ day from a baseline of 2,228 kcal/day for a sedentary $80 \mathrm{~kg}$ woman, with the goal of losing $8 \mathrm{~kg}$ in 6 months. This schedule was followed by a permanent reduction of energy intake to $2,178 \mathrm{kcal} /$ day to maintain the weight loss. By modifying the initial conditions of the model to represent an individual person, the simulator can be used for personalized goal setting and behavioral intervention planning. ${ }^{9}$

In reality, however, outpatient weight-loss interventions typically result in maximum weight loss after 6 to 8 months, followed by gradual weight regain over subsequent years. A common explanation for the weight loss plateau at 6 to 8 months is that energy expenditure decreases to match energy intake, pausing further weight loss (metabolic compensation). Weight regain occurs as individuals gradually weaken compliance with the diet that has resulted in weight loss (behavioral compensation). ${ }^{24}$

Hall et al. ${ }^{8}$ investigated the change of energy intake required to simulate a typically-observed, weight-loss and regain trajectory using a mathematical model. The results demonstrated that the observed weight change could have occurred if the subjects initially reduced their energy intake by about $800 \mathrm{kcal} /$ day, which was maintained for only 6 weeks. Energy intake gradually increased and returned to the original weight-maintenance level in the first year and was maintained there for the remaining 3-year simulation. The simulated TEE showed that negative energy balance was achieved for less than 8 months, after which positive energy balance and weight regain occurred. ${ }^{25}$

This predicted pattern of energy intake means that weight loss continues for several months at the same time that average energy intake slowly increases. The dieter may then misconstrue that maintenance of lifestyle change is not essential for continuing weight loss when weight regain has already begun. Even if the original lifestyle is resumed within the first year, the weight gain will gradually recur over many years because weight change occurs slowly. ${ }^{8}$ 
$\mathrm{NIH}\rangle$

National Institute of Diabetes and Digestive and Kidney Diseases

(i) Body Weight Planner I Balancing Your Food and Activity

\begin{tabular}{|c|c|c|}
\hline Starting Information & \multicolumn{2}{|c|}{ Advanced Controis: OFF } \\
\hline Weight & 80 & $\mathrm{Kg} \vee$ \\
\hline Sex & \multicolumn{2}{|l|}{ Female } \\
\hline Age & 50 & yrs \\
\hline Height & 160 & $\mathrm{~cm} \quad V$ \\
\hline \multirow{2}{*}{$\begin{array}{l}\text { Physical } \\
\text { Activity Level }\end{array}$} & \multicolumn{2}{|c|}{1.6} \\
\hline & \multicolumn{2}{|c|}{ Esilmate Your Level } \\
\hline
\end{tabular}
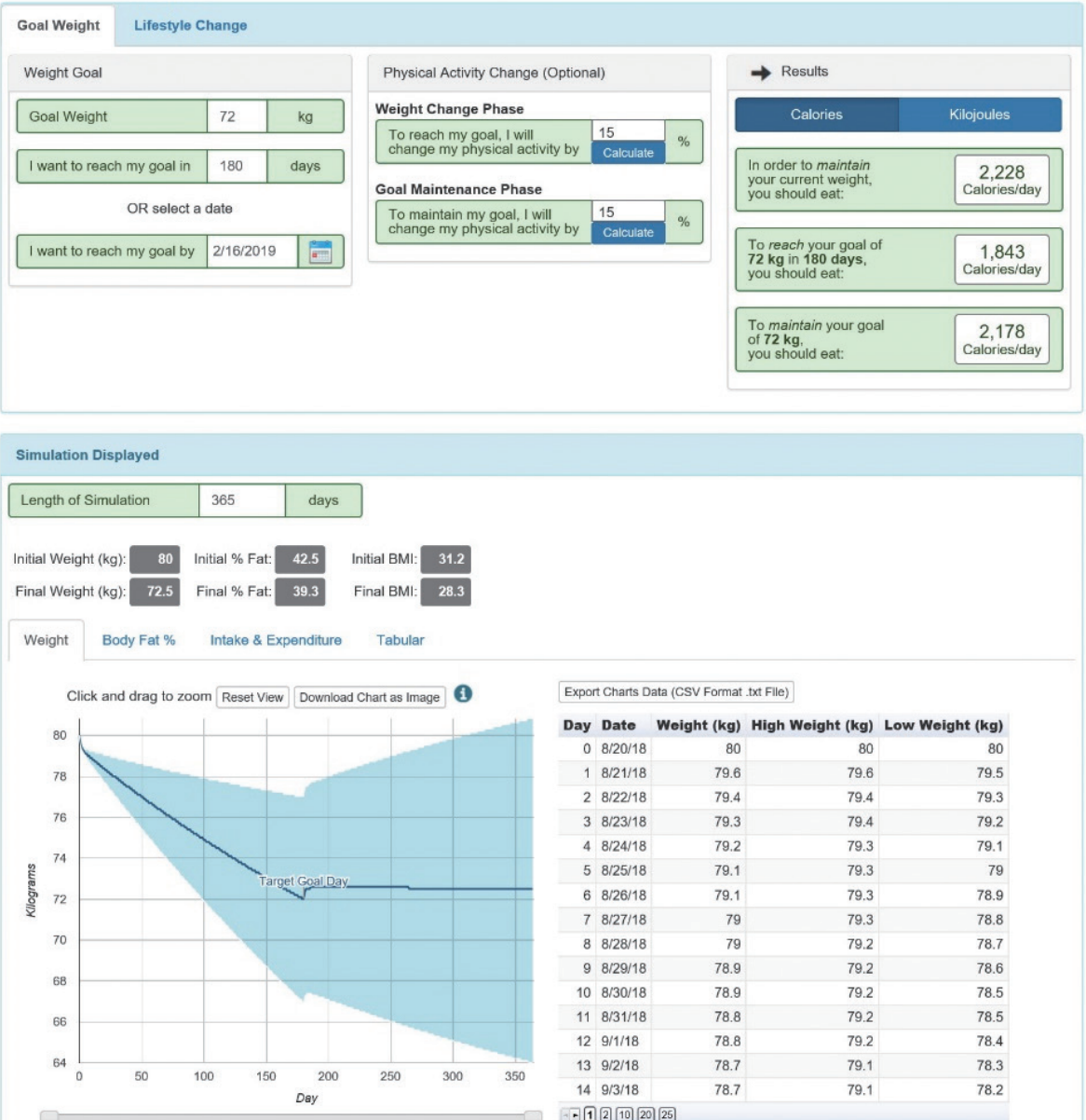

\begin{tabular}{|c|c|c|c|c|}
\hline \multicolumn{5}{|c|}{ Export Charts Data (CSV Format txt Flle) } \\
\hline Day & Date & Woight (kg) & High Woight (kg) & Low Weight (kg) \\
\hline 0 & 8/20/18 & 80 & 80 & 80 \\
\hline 1 & $8 / 21 / 18$ & 79.6 & 79.6 & 79.5 \\
\hline 2 & $8 / 22 / 18$ & 79.4 & 79.4 & 79.3 \\
\hline 3 & $8 / 23 / 18$ & 79.3 & 79.4 & 79.2 \\
\hline 4 & $8 / 24 / 18$ & 79.2 & 79.3 & 79.1 \\
\hline 5 & $8 / 25 / 18$ & 79.1 & 79.3 & 79 \\
\hline 6 & 8/26/18 & 79.1 & 79.3 & 78.9 \\
\hline 7 & $8 / 27 / 18$ & 79 & 79.3 & 78.8 \\
\hline 8 & $8 / 28 / 18$ & 79 & 79.2 & 78.7 \\
\hline 9 & 8/29/18 & 78.9 & 79.2 & 78.6 \\
\hline 10 & $8 / 30 / 18$ & 78.9 & 79.2 & 78.5 \\
\hline 11 & $8 / 31 / 18$ & 78.8 & 79.2 & 78.5 \\
\hline 12 & $9 / 1 / 18$ & 78.8 & 79.2 & 78.4 \\
\hline 13 & 9/2/18 & 78.7 & 79.1 & 78.3 \\
\hline 14 & $9 / 3 / 18$ & 78.7 & 79.1 & 78.2 \\
\hline
\end{tabular}

Figure 2. Web-based simulations ${ }^{9}$ for setting goals for weight loss and maintenance of reduced weight. The panel located on the top-left part of the simulator window specifies the baseline characteristics of the individual person or population average values. This example illustrates weight-related information for an 80-kg, 160-cm-tall, and 50-year-old woman. The top-middle panel specifies the goal weight (72 kg) and desired time interval to achieve the goal (180 days). The simulation displays the required changes of dietary energy intake to meet the goal and maintain the weight change. The simulated body weight trajectory is graphically displayed in the lower panel. Users can also modify physical activity to examine how the combination of diet and exercise interventions can achieve the same goal.

Estimation for obesity prevention at the population level

As mentioned earlier, energy intake and expenditure are tightly coupled over prolonged time intervals in adults living independently. The average daily excess of total energy intake over TEE, which is needed to create weight gain over a period of time, is referred to as the "energy imbalance gap." Estimating from 1978 to 2005 U.S. National Health and Nutrition Examination Survey data, the persistent daily energy imbalance between average daily energy intake and expenditure rate was about $7 \mathrm{kcal} / \mathrm{day}$ (or $30 \mathrm{~kJ}$ per day). This is equivalent to an average increase of energy stored in body fat and lean tissue divided by the time needed to store the energy. ${ }^{8}$ A small but chronic daily energy imbalance gap has caused the continuing weight gain observed at the population level. As these changes have persisted for the last three decades, the obesity epidemic has become a global problem.

In contrast to the small energy imbalance gap, the "maintenance energy gap" is defined as the increased average energy intake needed to sustain increased weight. The maintenance energy gap estimates the increased energy intake needed to maintain higher average weights following the obesity epidemic. Table 1 shows data 
Table 1. Trends of average weight, prevalence of obesity, and energy intake among Korean adult men aged over 19 years from 2007 to 2016

\begin{tabular}{|c|c|c|c|c|c|c|c|c|c|c|}
\hline \multirow{2}{*}{ Variable } & \multicolumn{10}{|c|}{ KNHANES } \\
\hline & 2007 & 2008 & 2009 & 2010 & 2011 & 2012 & 2013 & 2014 & 2015 & 2016 \\
\hline Mean body weight (kg) & 69.7 & 69.7 & 70 & 70.2 & 70.3 & 70.8 & 70.8 & 71 & 71.9 & 72.1 \\
\hline Prevalence of obesity $(\%)^{*, t}$ & 36.2 & 35.3 & 35.8 & 36.4 & 35.1 & 36.3 & 37.7 & 37.8 & 39.7 & 42.3 \\
\hline Total energy intake $(\mathrm{kcal})^{*}$ & $2,196.6$ & $2,216.7$ & $2,281.0$ & $2,468.4$ & $2,481.8$ & $2,391.9$ & $2,466.1$ & $2,442.0$ & $2,503.0$ & $2,426.8$ \\
\hline
\end{tabular}

*Age-standardized values were calculated using the age- and sex-specific structures of the estimated population in the 2005 Korea Census; ${ }^{\dagger} 0$ besity was defined as body mass index of $25 \mathrm{~kg} / \mathrm{m}^{2}$ or higher.

KNHANES, Korea National Health and Nutrition Examination Survey.

from the 2007-2016 Korea National Health and Nutrition Examination Surveys (KNHANES). Daily energy intake among men increased from 2,196 kcal in 2007 to 2,426 kcal in 2016, a maintenance energy gap of $230 \mathrm{kcal} /$ day over a period of 10 years among Korean adult men. Swinburn et al. ${ }^{26}$ estimated that a $10 \%$ higher total energy intake would equate to a $7 \%$ higher body weight using regression models for 1,399 adults with TEE from double-labeled water studies. Applying this result to the KNHANES data, the energy maintenance gap of $230 \mathrm{kcal}$ since 2006 would be predicted to result in a weight gain of $4.8 \mathrm{~kg}$. In reality, the average weight gain of men was only $2.3 \mathrm{~kg}$, but the prevalence of obesity increased by $6 \%$ during this period. This is a substantial change and shows that the reversal of obesity would require substantially large changes in terms of energy balance.

Prevention of further average excess weight gain can be accomplished with relatively slight changes (energy imbalance gap, about $7 \mathrm{kcal} /$ day). However, population weight has been accumulating for decades in most countries, and higher weights require greater energy intake to maintain. Thus, the difference between the energy required to stop weight gain and that required to reduce a specified amount of excess weight — the much larger maintenance energy gap-should be addressed. ${ }^{27}$ By applying the dynamic energy balance model to the U.S. population data and estimating the energy expenditure to reduce the prevalence of obesity, a negative energy balance of $24 \mathrm{kcal} /$ day (i.e., $100 \mathrm{~kJ} /$ day) will lead to a weight change of $1 \mathrm{~kg}$, and it will take about 1 year to achieve half of the total weight change. ${ }^{8}$ It is important to note that much larger changes are needed for obese individuals to return to their normal body weight. Thus, obesity prevention should be given priority, considering the limited effects of behavioral and pharmacological measures to lose weight.

The U.S. Healthy People 2020 objectives include reducing obe- sity prevalence among adults aged 20 years and older from $33.9 \%$ in $2005-2008$ to $30.5 \%$ by 2020 . A simulation study suggested that an $8.5 \%$ decrease from current daily calorie consumption (i.e., from $1,958 \mathrm{kcal}$ to $1,792 \mathrm{kcal}$ ) or a $7.5 \%$ increase in daily exercise (i.e., 29 minutes more day/person) would be needed to reach the target. However, the same researchers concluded that no single population-level intervention would achieve the Healthy People 2020 objective if implemented alone. For example, community-based moderate- to high-intensity exercise programs with dietary counseling could only reduce the energy intake by $1.2 \%$ and increase physical activity by $1.6 \%{ }^{28}$ This suggests the need for more long-term population intervention trials that combine several interventions, considering the disparity in obesity prevalence among populations. Recently, the Korean Ministry of Health and Welfare announced its national comprehensive measures for obesity management and proposed to continue curbing the growth of the obesity rate to maintain the figure at the 2016 level of $34.8 \%$ from the estimated obesity rate of $41.5 \%$ in 2022 . It must be verified if this goal is feasible with the current available interventions.

Young children are the top priority for obesity prevention; they have the smallest energy gaps to change and could be the first population to show evidence in reversing the epidemic. ${ }^{27}$ Preventive interventions against obesity in children have been shown to be effective $^{29}$ and more cost-effective than clinical interventions to treat obesity (Table 2). In particular, two interventions have attracted attention for cost-savings within a 10 -year period in the United States: an excise tax on sugar-sweetened beverages and elimination of the tax subsidy for TV advertising directed at children for nutritionally poor foods and beverages. ${ }^{30}$ These interventions are characterized by high feasibility, broad population reach, and relatively low cost. Excise and sales taxes on sugar-sweetened beverages are already in place in over 20 countries, and the effects are under investigation. ${ }^{31}$ 
Table 2. Estimated 1-year cost and outcomes of childhood obesity interventions

\begin{tabular}{|c|c|c|}
\hline Intervention & Change in BMl per person & Cost $(\$)$ \\
\hline Motivational interviewing intervention to modify nutrition and TV viewing habits 32 & $-0.21(-0.50$ to 0.07$)$ & $196^{*}$ \\
\hline Family-based diet and activity education ${ }^{33}$ & $-2.00(-3.6 \text { to } 3.4)^{\dagger}$ & $1,629^{\ddagger}$ \\
\hline Sugar sweetened beverage excise tax for ages $2-19$ years $\mathrm{s}^{30}$ & $-0.16(-0.37$ to -0.06$)$ & $8.54^{\S}$ \\
\hline Reduce tax subsidy of TV advertising ${ }^{30}$ & $-0.03(-0.05$ to -0.01$)$ & $1.16^{\S}$ \\
\hline Make early child educational settings healthier by increasing physical activity, improving nutrition, and reducing screen time ${ }^{30}$ & $-0.02(-0.04$ to -0.01$)$ & $57.8^{\S}$ \\
\hline State policy for active physical education ${ }^{30}$ & $-0.02(-0.05$ to -0.003$)$ & $401^{\S}$ \\
\hline
\end{tabular}

Values are presented as mean (range).

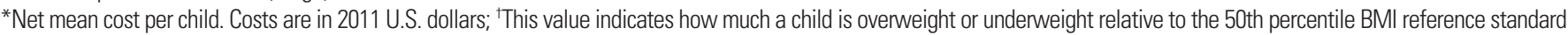
specific to his or her age and sex and was calculated by the following equation: [(child BMI-BMl at the 50th percentile)/BMl at the 50th percentile] $\times 100$; ${ }^{\prime}$ Total societal cost per family. Costs are in 2013 U.S. dollars; ${ }^{\S}$ Cost per unit BMI reduction. Costs are in 2014 U.S. dollars.

$\mathrm{BMI}$, body mass index.

\section{CONCLUSION}

Dynamic energy balance can effectively explain body weight change following negative energy balance. A change in energy intake influences energy expenditure. Whether diet-induced or exercise-driven, weight loss leads to a decrease in TEE, REE, and nonREE. Mechanisms associated with reduction of TEE following weight loss are likely to be related to decreased body mass and enhanced metabolic efficiency. Reducing calorie intake results in a decrease in body weight, followed by strong physiological adaptations to regain weight. Changes in body composition occur differently with elapsed time after the negative energy balance is achieved. During the first several weeks of calorie restriction, rapid weight loss occurs due to the combined effects of glycogen, protein, and fluid loss. With ongoing negative energy balance, fat mass decreases slowly due to the slower rates.

To avoid a typically observed weight-loss and regain trajectory, realistic weight loss goals should be established and maintained for more than 1 year. Using a mathematical model can help clinicians provide individuals advice about diet control. By modifying the initial conditions of the model to represent an individual person, the simulator can be used for personalized goal setting and behavioral intervention planning. It is important to emphasize steady efforts to maintain reduced weight over efforts to lose weight.

Although energy intake and expenditure are tightly coupled over prolonged intervals in adults living independently, small but chronic daily energy imbalance gap changes (about $7 \mathrm{kcal} /$ day) have caused the continuing weight gain seen at the population level. ${ }^{8}$ Epidemiologic data show that Korean adult men consume $230 \mathrm{kcal}$ more per day compared to 10 years ago, thereby increasing their average weight by $2.3 \mathrm{~kg}$, while obesity prevalence increased by $6 \%$. This is a substantial change and reveals that reversal of obesity requires large changes in terms of energy balance.

Because obesity is difficult to reverse, obesity prevention must be prioritized, along with implementation of obesity prevention strategies with high feasibility, broad population reach, and relatively low cost for young children, who have the smallest energy gaps to change. It is difficult to reduce the rapidly increasing obese population with only one strategy. Therefore, it is necessary to establish a complex approach to prevent obesity by paying attention to the results of practical application of several strategies shown to be successful through cost-effectiveness analysis.

\section{CONFLICTS OF INTEREST}

The author declares no conflict of interest.

\section{ACKNOWLEDGMENTS}

This work was supported by a grant from the 2017 research year of Inje University (grant No. 20170047) and was conducted at the Sheikh Khalifa Specialty Hospital in the United Arab Emirates.

\section{REFERENCES}

1. GBD 2015 Obesity Collaborators, Afshin A, Forouzanfar MH, Reitsma MB, Sur P, Estep K, et al. Health effects of overweight and obesity in 195 countries over 25 years. N Engl J Med 2017; 
377:13-27.

2. NCD Risk Factor Collaboration (NCD-RisC). Trends in adult body-mass index in 200 countries from 1975 to 2014: a pooled analysis of 1698 population-based measurement studies with 19.2 million participants. Lancet 2016;387:1377-96.

3. Korean Society for the Study of Obesity. 2018 Clinical practice guidelines for overweight and obesity. Seoul: Committee of Clinical Guideline, Korean Society for the Study of Obesity; 2018.

4. Guth E. JAMA patient page: healthy weight loss. JAMA 2014; 312:974.

5. Shils ME, Shike M, Ross AC, Caballero B, Cousins RJ. Modern nutrition in health and disease. Philadelphia (PA): Lippincott Williams \& Wilkins; 2006.

6. Byrne NM, Meerkin JD, Laukkanen R, Ross R, Fogelholm M, Hills AP. Weight loss strategies for obese adults: personalized weight management program vs. standard care. Obesity (Silver Spring) 2006;14:1777-88.

7. Thomas DM, Martin CK, Lettieri S, Bredlau C, Kaiser K, Church T, et al. Can a weight loss of one pound a week be achieved with a 3500-kcal deficit? Commentary on a commonly accepted rule. Int J Obes (Lond) 2013;37:1611-3.

8. Hall KD, Sacks G, Chandramohan D, Chow CC, Wang YC, Gortmaker SL, et al. Quantification of the effect of energy imbalance on bodyweight. Lancet 2011;378:826-37.

9. National Institute of Diabetes and Digestive and Kidney Diseases. Body weight planner [Internet]. Bethesda, MD: National Institute of Diabetes and Digestive and Kidney Diseases; 2011 [cited 2018 Aug 18]. Available from: https://www.niddk.nih.gov/bwp

10. Thomas DM, Ciesla A, Levine JA, Stevens JG, Martin CK. A mathematical model of weight change with adaptation. Math Biosci Eng 2009;6:873-87.

11. Pennington Biomedical Research Center. Weight loss predictor [Internet]. Baton Rouge, LA: Pennington Biomedical Research Center; 2009 [cited 2018 Aug 18]. Available from: http://www. pbrc.edu/research-and-faculty/calculators/weight-loss-predictor/

12. Müller MJ, Enderle J, Bosy-Westphal A. Changes in energy expenditure with weight gain and weight loss in humans. Curr
Obes Rep 2016;5:413-23.

13. Schwartz MW, Seeley RJ, Zeltser LM, Drewnowski A, Ravussin E, Redman LM, et al. Obesity pathogenesis: an endocrine society scientific statement. Endocr Rev 2017;38:267-96.

14. Bray GA, Flatt JP, Volaufova J, Delany JP, Champagne CM. Corrective responses in human food intake identified from an analysis of 7-d food-intake records. Am J Clin Nutr 2008;88: 1504-10.

15. Roser M, Ritchie H. Food per person [Internet]. Oxford: Our World in Data; 2017 [cited 2018 Nov 17]. Available from: https:// ourworldindata.org/food-per-person

16. Benton D, Young HA. Reducing calorie intake may not help you lose body weight. Perspect Psychol Sci 2017;12:703-14.

17. Speakman JR, Levitsky DA, Allison DB, Bray MS, de Castro JM, Clegg DJ, et al. Set points, settling points and some alternative models: theoretical options to understand how genes and environments combine to regulate body adiposity. Dis Model Mech 2011;4:733-45.

18. Greenway FL. Physiological adaptations to weight loss and factors favouring weight regain. Int J Obes (Lond) 2015;39: 1188-96.

19. Heymsfield SB, Thomas D, Nguyen AM, Peng JZ, Martin C, Shen W, et al. Voluntary weight loss: systematic review of early phase body composition changes. Obes Rev 2011;12:e348-61. 20. Müller MJ, Enderle J, Pourhassan M, Braun W, Eggeling B, Lagerpusch M, et al. Metabolic adaptation to caloric restriction and subsequent refeeding: the Minnesota Starvation Experiment revisited. Am J Clin Nutr 2015;102:807-19.

21.Johannsen DL, Knuth ND, Huizenga R, Rood JC, Ravussin E, Hall KD. Metabolic slowing with massive weight loss despite preservation of fat-free mass. J Clin Endocrinol Metab 2012; 97:2489-96.

22. Fothergill E, Guo J, Howard L, Kerns JC, Knuth ND, Brychta $\mathrm{R}$, et al. Persistent metabolic adaptation 6 years after "The Biggest Loser” competition. Obesity (Silver Spring) 2016;24: 1612-9.

23. Hall KD. Diet versus exercise in "the biggest loser" weight loss competition. Obesity (Silver Spring) 2013;21:957-9.

24.Dhurandhar EJ, Kaiser KA, Dawson JA, Alcorn AS, Keating $\mathrm{KD}$, Allison DB. Predicting adult weight change in the real 
world: a systematic review and meta-analysis accounting for compensatory changes in energy intake or expenditure. Int $\mathrm{J}$ Obes (Lond) 2015;39:1181-7.

25. Hall KD. Predicting metabolic adaptation, body weight change, and energy intake in humans. Am J Physiol Endocrinol Metab 2010;298:E449-66.

26. Swinburn BA, Sacks G, Lo SK, Westerterp KR, Rush EC, Rosenbaum M, et al. Estimating the changes in energy flux that characterize the rise in obesity prevalence. Am J Clin Nutr 2009;89:1723-8.

27. Gortmaker SL, Swinburn BA, Levy D, Carter R, Mabry PL, Finegood DT, et al. Changing the future of obesity: science, policy, and action. Lancet 2011;378:838-47.

28. Basu S, Seligman H, Winkleby M. A metabolic-epidemiological microsimulation model to estimate the changes in energy intake and physical activity necessary to meet the Healthy People 2020 obesity objective. Am J Public Health 2014;104: 1209-16.

29. Kim S, Sung E, Yoo S. Evidence of interventions for prevent- ing obesity of children and adolescents using existing systematic reviews and meta-analyses. Korean J Health Promot 2016; 16:231-50.

30. Gortmaker SL, Long MW, Resch SC, Ward ZJ, Cradock AL, Barrett JL, et al. Cost effectiveness of childhood obesity interventions: evidence and methods for CHOICES. Am J Prev Med 2015;49:102-11.

31. Silver LD, Ng SW, Ryan-Ibarra S, Taillie LS, Induni M, Miles $\mathrm{DR}$, et al. Changes in prices, sales, consumer spending, and beverage consumption one year after a tax on sugar-sweetened beverages in Berkeley, California, US: a before-and-after study. PLoS Med 2017;14:e1002283.

32. Wright DR, Taveras EM, Gillman MW, Horan CM, Hohman $\mathrm{KH}$, Gortmaker SL, et al. The cost of a primary care-based childhood obesity prevention intervention. BMC Health Serv Res 2014;14:44.

33. Quattrin T, Cao Y, Paluch RA, Roemmich JN, Ecker MA, Epstein LH. Cost-effectiveness of family-based obesity treatment. Pediatrics 2017;140:e20162755. 